\title{
Electronic structure of high-temperature superconductors
}

\author{
Brent A. Richert and Roland E. Allen \\ Center for Theoretical Physics, Department of Physics, Texas A \& M University, \\ College Station, Texas 77843 \\ (Received 20 August 1987; revised manuscript received 30 November 1987)
}

\begin{abstract}
The electronic energy bands, local densities of states, and valences have been calculated for $\mathrm{La}_{1.85} \mathrm{Sr}_{0.15} \mathrm{CuO}_{4}$ and $\mathrm{YBa}_{2} \mathrm{Cu}_{3} \mathrm{O}_{7}$, with a tight-binding model fitted to previous calculations for $\mathrm{La}_{2} \mathrm{CuO}_{4}$. Strong $d$-state peaks, predicted about $5 \mathrm{eV}$ above the Fermi energy for La, $\mathrm{Y}$, and $\mathrm{Ba}$, should be observable in inverse photoemission, electron energy-loss spectroscopy, and final-state photoemission experiments.
\end{abstract}

Since the discovery of high-temperature superconductivity, ${ }^{1,2}$ there has been rapid experimental progress. As yet, however, there is no confirmed theory. Here we present calculations of the electronic structures of $\mathrm{La}_{1.85} \mathrm{Sr}_{0.15} \mathrm{CuO}_{4}$ and $\mathrm{YBa}_{2} \mathrm{Cu}_{3} \mathrm{O}_{7}$ in the one-electron approximation, using a simple tight-binding Hamiltonian.

The band structures of $\mathrm{La}_{2} \mathrm{CuO}_{4}$ and $\mathrm{YBa}_{2} \mathrm{Cu}_{3} \mathrm{O}_{7}$ have been previously calculated by Mattheiss and Hamann, ${ }^{3,4}$ Freeman and co-workers, ${ }^{5,6}$ Oguchi, ${ }^{7}$ and Takegahara, Harima, and Yanase. ${ }^{8}$ As will be seen below, we find that a tight-binding model ${ }^{9}$ very nearly reproduces the results of these more complicated calculations, provided that one includes the unoccupied $d$ states on $\mathrm{La}, \mathrm{Y}$, and $\mathrm{Ba}$. We also find that the tight-binding parameters are transferable from the La compound to the 1:2:3 material. The advantage of a simple tight-binding description is that it provides a convenient starting point for further theoretical studies in various directions, such as models of superconductivity ${ }^{10,11}$ and calculations of material properties. ${ }^{12,13}$

The model employed here includes the valence $p$ and $s$ orbitals of $\mathrm{O}$ and the $d$ and $s$ orbitals of all the metal atoms. There are thus 34 orbitals per chemical unit for $\mathrm{La}_{2} \mathrm{CuO}_{4}$ and 64 for $\mathrm{YBa}_{2} \mathrm{Cu}_{3} \mathrm{O}_{7}$. Since the $d$ states of $\mathrm{La}, \mathrm{Y}$, and $\mathrm{Ba}$ are unoccupied, one is tempted to neglect them. However, they appear to be required for a good description of charge transfer. (We find that $\mathrm{La}, \mathrm{Y}$, and Ba lose substantially more electronic charge when their $d$ states are neglected.) On the other hand, the $f$ states of $\mathrm{La}, \mathrm{Ba}$, and $\mathrm{Y}$ appear to be relatively unimportant, ${ }^{8}$ so they are omitted. It appears to be a good general principle that the accuracy of a tight-binding model is improved if unoccupied $d$ states near the Fermi energy are included. ${ }^{14}$

The "atomic energies" $\varepsilon_{s}, \varepsilon_{p}$, and $\varepsilon_{d}$ for $\mathrm{O}, \mathrm{La}, \mathrm{Sr}, \mathrm{Y}$, and $\mathrm{Ba}$ were simply taken from the table of Harrison, ${ }^{15}$ with $\varepsilon_{d}$ taken to have the same value for $\mathrm{Y}$ and $\mathrm{Sr}$ and for $\mathrm{La}, \mathrm{Ba}$, and $\mathrm{Lu}$. The interatomic matrix elements $V_{s s \sigma}$, etc., together with $\varepsilon_{s}$ and $\varepsilon_{d}$ for $\mathrm{Cu}$, were determined by fitting the band structures of Refs. 3, 5, 7, and 8 for $\mathrm{La}_{2} \mathrm{CuO}_{4}$, and were then carried over to $\mathrm{YBa}_{2} \mathrm{Cu}_{3} \mathrm{O}_{7}$ without modification. The interatomic matrix elements are defined in Ref. 15 as

$$
V_{l l^{\prime} m}=\eta_{l l^{\prime} m} \frac{\hbar^{2}}{m_{e} d^{2}}\left(l, l^{\prime}=s \text { or } p\right)
$$

and

$$
V_{l d m}=\eta_{l d m} \frac{\hbar^{2} r d^{3 / 2}}{m_{e} d^{7 / 2}}(l=s \text { or } p) .
$$

The matrix element varies with the indicated inverse power of the interatomic distance $d$, and is characterized by a strength $\eta$. The size of the $d$ orbital interaction is characterized by $r_{d}$. We obtain $\eta_{s s \sigma}=-1.1, \eta_{s p \sigma}=0.9$, $\eta_{s d \sigma}=-1.6, \eta_{p d \sigma}=-2.5$, and $\eta_{p d x}=1.4$; also $r_{d}=0.95 \AA$ for $\mathrm{Cu}$ and $1.6 \AA$ for the other metal atoms. (Since $r_{d}$ for the other metal atoms has a relatively minor effect, we simply retained the value of Ref. 15 , and adjusted $r_{d}$ only for $\mathrm{Cu}$.) The remaining parameters are listed in Table $\mathrm{I}$. The replacement $\mathrm{La}_{2} \rightarrow \mathrm{La}_{2}-x \mathrm{Sr}_{x}$ was made in the virtual crystal approximation, i.e., by simply letting $\varepsilon_{s}(\mathrm{La}) \rightarrow(1-x / 2) \varepsilon_{s}(\mathrm{La})+(x / 2) \varepsilon_{s}(\mathrm{Sr})$. Since $\varepsilon_{s}(\mathrm{Sr})$ $\approx \varepsilon_{s}(\mathrm{La})$, this is a good approximation in the present context. It also follows that the electronic states are virtually identical to those of $\mathrm{La}_{2} \mathrm{CuO}_{4}$ for the same crystal structure, and the only effect of the $\mathrm{Sr}$ is to remove $x$ electrons per chemical unit.

We originally included second-neighbor interactions, such as $\mathbf{O}-\mathrm{O}$, but found that the optimum value of each such interaction was zero. This is fortunate, since a nearest-neighbor model is much more convenient in most applications.

The crystal structure of $\mathrm{La}_{1.85} \mathrm{Sr}_{0.15} \mathrm{CuO}_{4}$ was taken from experiment, ${ }^{16}$ but the doubling of the unit cell and small orthorhombic distortion were ignored for simplicity, as in Refs. 3, 5, 7, and 8. (It is not hard to perform the calculations for a larger or orthorhombic unit cell, but the band structure of Fig. 1 would have a complicated appearance. The small displacements associated with the distortions below about $200 \mathrm{~K},{ }^{16}$ although fundamentally significant, would have an imperceptible effect in the

TABLE I. "Atomic energies" in eV.

\begin{tabular}{lcccccc}
\hline & $\mathrm{Cu}$ & $\mathrm{O}$ & $\mathrm{La}$ & $\mathrm{Sr}$ & $\mathrm{Y}$ & $\mathrm{Ba}$ \\
\hline$\varepsilon_{d}\left(\varepsilon_{p}\right)$ & -14 & $(-14)$ & -6.6 & -6.8 & -6.8 & -6.6 \\
$\varepsilon_{s}$ & -12 & -29 & -4.9 & -5.0 & -5.5 & -4.5 \\
\hline \hline
\end{tabular}


present calculations.) The orthorhombic crystal structure of $\mathrm{YBa}_{2} \mathrm{Cu}_{3} \mathrm{O}_{7}$ was taken from Ref. 16.

Our calculated energy bands for $\mathrm{La}_{1.85} \mathrm{Sr}_{0.15} \mathrm{CuO}_{4}$ are shown in Fig. 1, with the conventional labeling of the symmetry points ${ }^{3,17}$ and the zero of energy shifted to $E_{F}$. Comparison with the previous calculations ${ }^{3,5,7,8}$ shows the same general behavior. The valence bandwidths at the $\Delta U$ midpoint and at $X$ are 7.0 and $9.7 \mathrm{eV}$, compared with 6.5 and $9.4 \mathrm{eV}$ in Ref. 3. The single prominent $\mathrm{Cu}(d)-\mathrm{O}(p)$ antibonding state protruding above $E_{F}$ at $X$ overlaps the lowest-lying conduction band by $0.9 \mathrm{eV}$, compared with $1.0 \mathrm{eV}$ in Refs. 3 and 5, and $0.6 \mathrm{eV}$ in Ref. 8. There are, of course, minor differences; for example, the highest occupied states along the line $\Delta U$ are about $0.5 \mathrm{eV}$ nearer $E_{F}$ in the more complicated calculations, but the most important features are in remarkably good agreement. The bands are found to have almost two-dimensional character, ${ }^{3,5}$ with little variation in the $k_{z}$ direction, perpendicular to the $\mathrm{CuO}_{2}$ planes.

Figure 2 shows the local densities of states for this material, with $O(1)$ and $O(2)$ respectively labeling the inplane and out-of-plane oxygen sites. (The calculation employed 24 special points ${ }^{18}$ in the irreducible part of the Brillouin zone, with a $0.2 \mathrm{eV}$ broadening.) The total density of states at $E_{F}$ is 1.2 states/eV cell, compared with previous calculations ranging from 1.3 states $/ \mathrm{eV}$ cell ${ }^{3}$ to 2.1 states/eV cell. ${ }^{7}$ Since $\mathrm{O}(2)$ is more weakly bonded to $\mathrm{Cu}$, it is more ionic, with a sharper $p$ state peak below $E_{F}$. The $\mathrm{Cu}(d)$ and $\mathrm{O}(p)$ states, which have nearly the same energy, are almost fully occupied. The electron occupancy $n_{i}$ for each atomic site $i$ is determined by a weighted average over the Brillouin zone sample points of

$$
n_{i}=2 \sum_{\alpha, E \leq E_{F}}\left|\psi_{i}^{\alpha}(E)\right|^{2},
$$

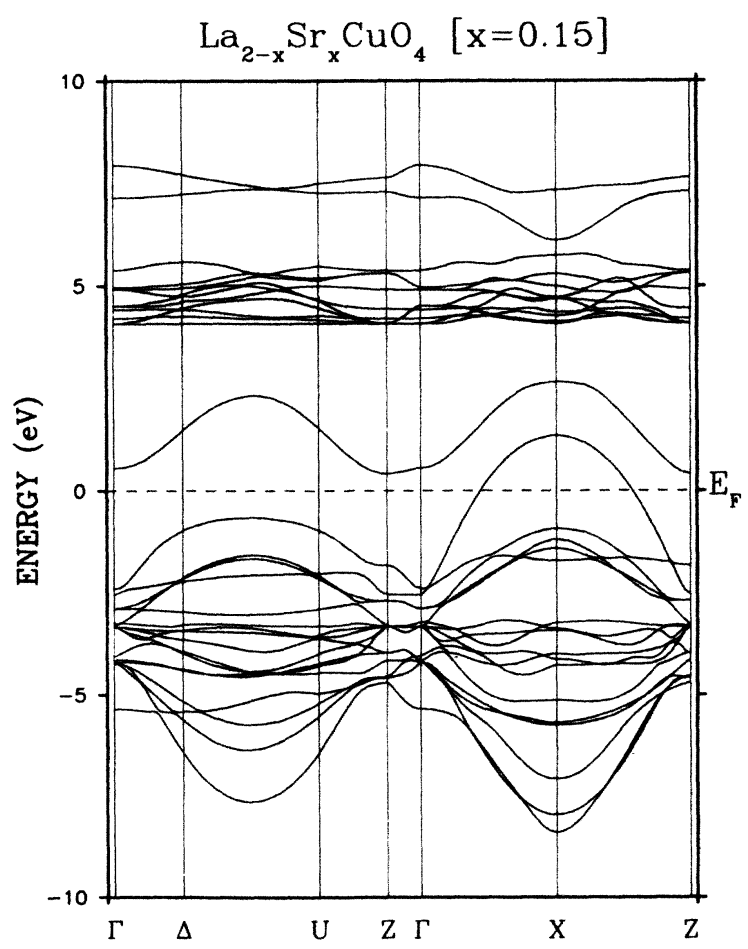

FIG. 1. Electronic energy bands for $\mathrm{La}_{1.85} \mathrm{Sr}_{0.15} \mathrm{CuO}_{4}$.
Density of States

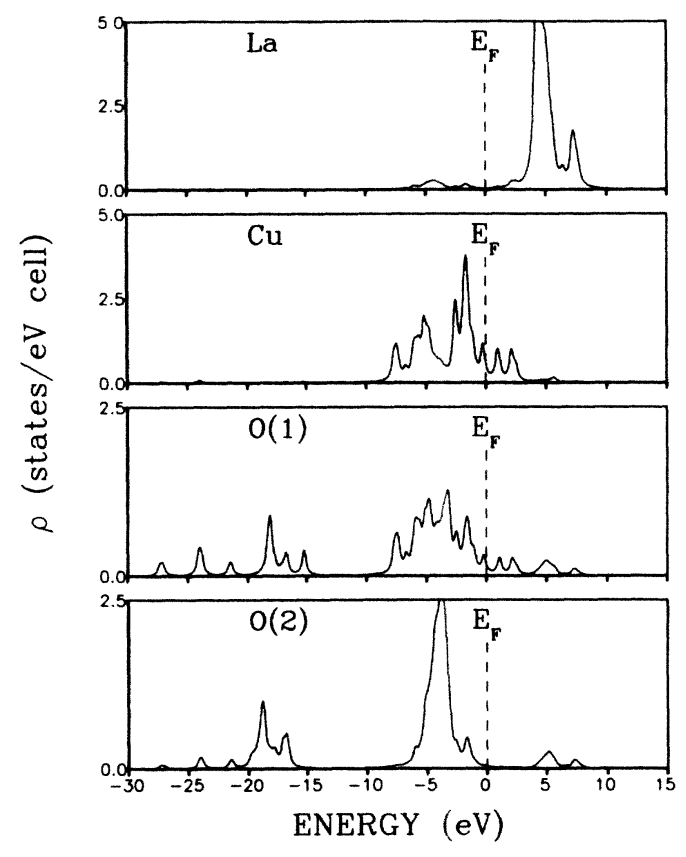

FIG. 2. Local densities of states for $\mathrm{La}_{1.85} \mathrm{Sr}_{0.15} \mathrm{CuO}_{4}$.

where $\psi_{i}^{a}(E)$ is the component of the eigenfunction corresponding to valence orbital $\alpha$ on atom $i$, and two electrons occupy each state up to $E_{F}$. We find that $\mathrm{Cu}$ has very nearly 10 electrons, $O(1)$ about 7.1 , and $O(2)$ about 7.5 . One might interpret the first number as corresponding to approximately zero $\mathrm{Cu}(d)$ holes.

If we define the valence $\Delta n$ to be the number of electrons lost by the atom when it is bonded in the solid, then $\Delta n=+1.0,-1.1$, and -1.5 for $\mathrm{Cu}, \mathrm{O}(1)$, and $\mathrm{O}(2)$, respectively, as indicated in Table II. Since all bonds have some covalent character, $\Delta n$ is expected to be substantially less than the customary formal valence of chemistry. For example, a valence calculation which we performed for cubic $\mathrm{Cu}_{2} \mathrm{O}$ (cuprite) gives a $\Delta n$ of +0.24 for $\mathrm{Cu}$ and -0.48 for $\mathrm{O}$, compared with the formal valences $\mathrm{Cu}^{1+}$ and $\mathrm{O}^{2-}$. Similarly, a calculation for monoclinic $\mathrm{CuO}$ (tenorite) yields $\mathrm{Cu}^{0.79+}$ and $\mathrm{O}^{0.79-}$, compared with the formal valences $\mathrm{Cu}^{2+}$ and $\mathrm{O}^{2-}$. If we were to interpret the formal valence as roughly twice the calculated valence $\Delta \boldsymbol{n}$ (up to the maximum reasonable value), then Table II

TABLE II. Valence $\Delta n$.

\begin{tabular}{|c|c|c|c|}
\hline \multicolumn{2}{|c|}{$\mathrm{La}_{1.85} \mathrm{Sr}_{0.15} \mathrm{CuO}_{4}$} & \multicolumn{2}{|c|}{$\mathrm{YBa}_{2} \mathrm{Cu}_{3} \mathrm{O}_{7}$} \\
\hline \multirow[t]{2}{*}{$\mathrm{Cu}$} & +1.02 & $\mathrm{Cu}(2)$ & +0.98 \\
\hline & & $\mathrm{Cu}(1)$ & +1.34 \\
\hline \multirow[t]{3}{*}{$O(1)$} & -1.12 & $O(1)$ & -1.12 \\
\hline & & $O(2)$ & -1.09 \\
\hline & & $O(3)$ & -1.11 \\
\hline$O(2)$ & -1.51 & $O(4)$ & -1.21 \\
\hline $\mathrm{La}$ & +2.12 & Y & +1.77 \\
\hline $\mathrm{Sr}$ & +1.12 & $\mathbf{B a}$ & +1.44 \\
\hline
\end{tabular}




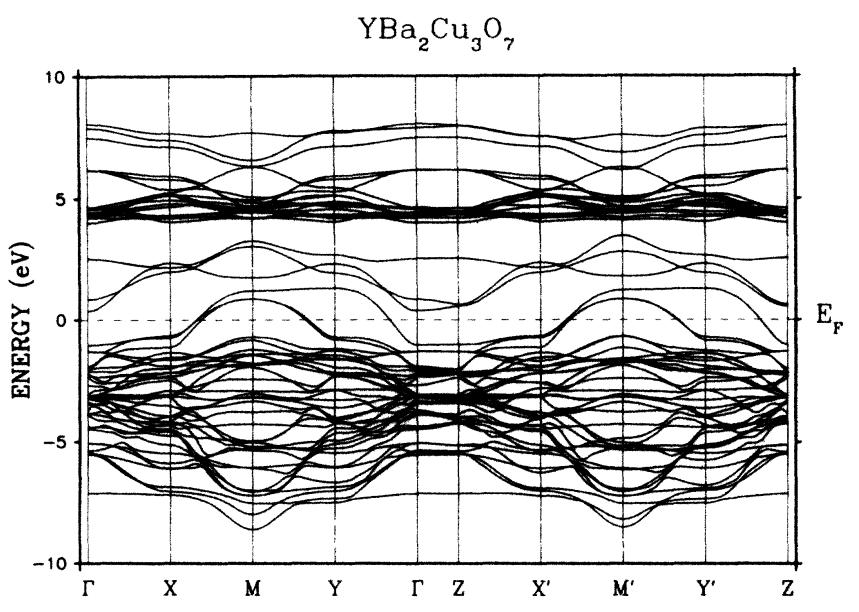

FIG. 3. Energy bands for $\mathrm{YBa}_{2} \mathrm{Cu}_{3} \mathrm{O}_{7}$.

gives $\mathrm{Cu}^{2+}, \mathrm{O}^{2-}, \mathrm{La}^{3+}$, and $\mathrm{Sr}^{2+}$. The exact balancing of these formal valences in $\mathrm{La}_{2} \mathrm{CuO}_{4}$, as in $\mathrm{SrTiO}_{3}$ and related materials, suggests a structural stability resulting largely from the charge transfer. ${ }^{15,16}$ The divalent nature of $\mathrm{Sr}$, of course, indicates the presence of holes in $\mathrm{La}_{2-x} \mathrm{Sr}_{x} \mathrm{CuO}_{4}$.

Figure 3 shows our calculated energy bands for $\mathrm{YBa}_{2} \mathrm{Cu}_{3} \mathrm{O}_{7}$, with the labeling of the symmetry points defined in Fig. 4. The valence bandwidths at $M$ and $M^{\prime}$ are 9.7 and $9.6 \mathrm{eV}$, compared with $8.9 \mathrm{eV}$ in Ref. 6. The bands protruding above $E_{F}$ at $M$ and $Y$ peak at 1.5 and $1.6 \mathrm{eV}$ above $E_{F}$, compared with 1.7 and $1.8 \mathrm{eV}$ in Ref. 6. The near equivalence of the left- and right-hand sides, and the small variation along $\Gamma Z$, demonstrate the two dimensionality of the electronic structure. ${ }^{4,6}$ For this triple perovskite structure, there are now three antibonding $\mathrm{Cu}(d)-\mathrm{O}(p)$ bands protruding above $E_{F}$ at $M$ and $M^{\prime}$. Two of them are nearly the same as the corresponding band for the La compound in Fig. 1, being associated with the $\mathrm{CuO}_{2}$ planes adjacent to the $\mathrm{Y}$; but one has essentially no dispersion along $M Y$, being associated with $\mathrm{CuO}$ chains in the $y$ direction.

The local densities of states for this 1:2:3 material are shown in Figs. 5 and 6 . (We used 64 special points in the

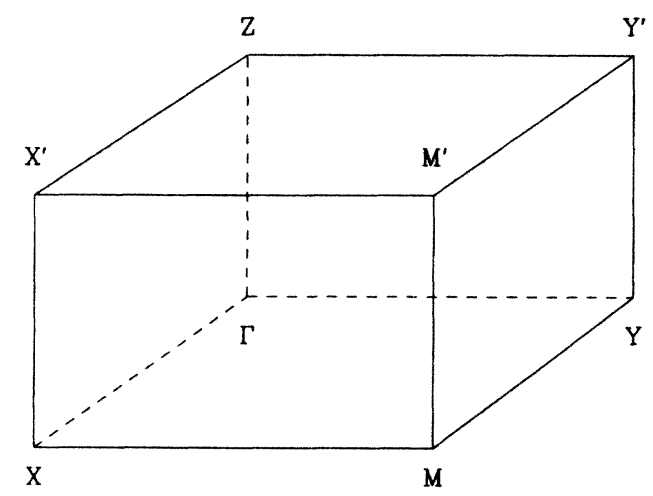

FIG. 4. Symmetry points of Fig. 3. $\Gamma Y$ is parallel to the $\mathrm{CuO}$ chains.

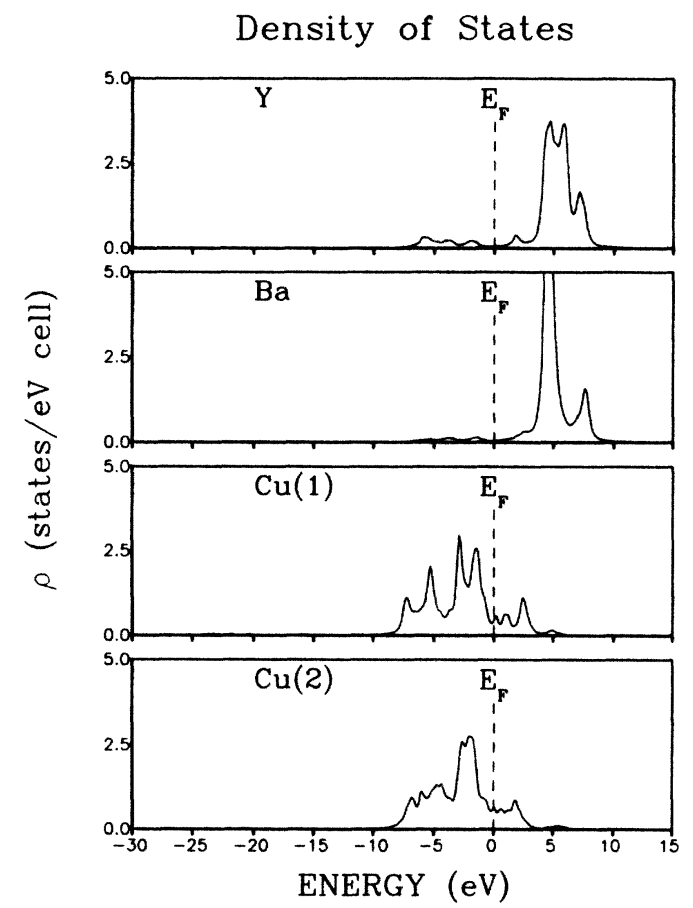

FIG. 5. Local densities of states for $\mathrm{YBa}_{2} \mathrm{Cu}_{3} \mathrm{O}_{7}$, metal atoms.

irreducible part of the Brillouin zone, and $0.2 \mathrm{eV}$ broadening.) The total density of states at $E_{F}$ is 3.2 states/eV cell, compared with 3.0 states/eV cell in Ref. 4 and 3.4 states/eV cell in Ref. 6 . $\mathrm{Cu}(1)$ and $\mathrm{O}(1)$ refer to the $\mathrm{CuO}$ chains; $\mathrm{Cu}(2), \mathrm{O}(2)$, and $\mathrm{O}(3)$ to $\mathrm{CuO}_{2}$ planes, as in Ref. 16 ; and $O(4)$ to the out-of-plane oxygens. The features

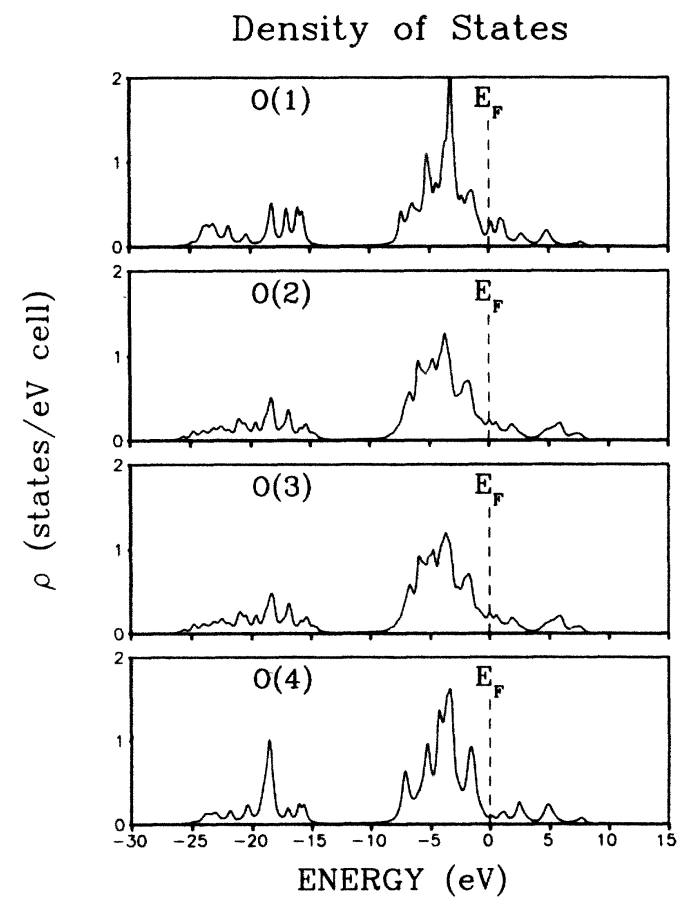

FIG. 6. Local densities of states for $\mathrm{YBa}_{2} \mathrm{Cu}_{3} \mathrm{O}_{7}$, oxygen atoms. 
are very similar to those for the La compound. For both materials, there is a rather low density of states at the Fermi energy. ${ }^{4,6}$ Strong $d$-state peaks are predicted a few $\mathrm{eV}$ above the Fermi energy for $\mathrm{Y}$ and $\mathrm{Ba}$ in $\mathrm{YBa}_{2} \mathrm{Cu}_{3} \mathrm{O}_{7}$, for $\mathrm{La} / \mathrm{Sr}$ in $\mathrm{La}_{2}{ }_{x} \mathrm{Sr}_{x} \mathrm{CuO}_{4}$, and for the corresponding metal atoms in related high-temperature superconductors. These peaks should be observable in electron energy-loss spectroscopy ${ }^{19}$ and final-state photoemission spectroscopy. ${ }^{20}$

The calculated valences $\Delta \boldsymbol{n}$ are again given in Table II. Notice that $\Delta n$ is approximately +1 for $\mathrm{Cu}$ in the $\mathrm{CuO}_{2}$ planes, just as in the La compound, but $\Delta n=+1.34$ for the $\mathrm{CuO}$ chains. One might interpret this result as corresponding to $\frac{1}{3} \mathrm{Cu}(d)$ hole per chemical unit with no doping. This difference in valences should be observable in photoemission on high-quality, single-crystal surfaces. If we again were to interpret the formal valence as very roughly twice $\Delta n$, Table II would give $\mathrm{Cu}^{2+}$ (planes), $\mathrm{Cu}^{3+}$ (chains), $\mathrm{O}^{2-}, \mathrm{Y}^{3+}$, and $\mathrm{Ba}^{2+}$, so that the formal valences again neatly cancel in $\mathrm{YBa}_{2} \mathrm{Cu}_{3} \mathrm{O}_{7}$.

In summary, we have calculated the electronic structures of the most typical members of the two known classes of high-temperature superconductors. The present results, obtained with a simple tight-binding model, are in good agreement with those obtained in more complicated calculations. ${ }^{3-8}$ A remarkably simple picture emerges, with conclusions that can be tested experimentally.

After the original submission of the present paper, three papers appeared with inverse photoemission measurements indicating unoccupied $d$ bands for both $\mathrm{La}_{1.85} \mathrm{Sr}_{0.15} \mathrm{CuO}_{4}$ and $\mathrm{YBa}_{2} \mathrm{Cu}_{3} \mathrm{O}_{7-y}$ which are in approximate agreement with those predicted here. ${ }^{21-23}$ Since the $f$-electron states have little influence on the other states and on the charge transfer, ${ }^{8}$ we have omitted them from the present model; the strong features associated with unoccupied $f$ states in the experimental spectra are consequently also omitted. Finally, we mention that Onellion, Margaritondo, and co-workers have observed the dispersion of the antibonding $\mathrm{Cu}-\mathrm{O}$ band along $\Gamma M$ for $\mathrm{YBa}_{2} \mathrm{Cu}_{3} \mathrm{O}_{7}{ }^{24}$

We thank the Office of Naval Research for their support, which made this work possible under Grant No. N00014-82-K-0447. Additional support was provided by the Robert A. Welch Foundation. B. A. R. was partially supported by the U.S. Air Force Institute of Technology.
${ }^{1}$ J. G. Bednorz and K. A. Müller, Z. Phys. B 64, 189 (1986).

${ }^{2}$ M. K. Wu, J. R. Ashburn, C. J. Torng, P. H. Hor, R. L. Meng, L. Gao, Z. J. Huang, Y. Q. Wang, and C. W. Chu, Phys. Rev. Lett. 58, 908 (1987).

${ }^{3}$ L. F. Mattheiss, Phys. Rev. Lett. 58, 1028 (1987).

${ }^{4}$ L. F. Mattheiss and D. R. Hamann, Solid State Commun. 63, 395 (1987).

5J. Yu, A. J. Freeman, and J.-H. Xu, Phys. Rev. Lett. 58, 1035 (1987).

${ }^{6}$ S. Massidda, J. Yu, A. J. Freeman, and D. D. Koelling, Phys. Lett. A 122, 198 (1987).

${ }^{7}$ T. Oguchi, Jpn. J. Appl. Phys. 26, L417 (1987).

${ }^{8}$ K. Takegahara, H. Harima, and A. Yanase, Jpn. J. Appl. Phys. 26, L352 (1987).

${ }^{9} \mathrm{An}$ earlier version of the present model for $\mathrm{La}_{1.85} \mathrm{Sr}_{0.15} \mathrm{CuO}_{4}$ was given in B. A. Richert and R. E. Allen, in Proceedings of the 18th International Conference on Low Temperature Physics, edited by Y. Nagaoka [Jpn. J. Appl. Phys. 26, Suppl. 26-3, 989 (1987)].

${ }^{10}$ B. A. Richert and R. E. Allen, in Proceedings of the 18th International Conference on Low Temperature Physics, edited by Y. Nagaoka [Jpn. J. Appl. Phys. 26, Suppl. 26-3, 2047 (1987)].

${ }^{11}$ B. A. Richert and R. E. Allen, in Proceedings of the Drexel International Conference on High Temperature Superconductivity, edited by S. Bose and S. Tyagi (World Scientific, Singapore, 1988), p. 149.

${ }^{12}$ B. A. Richert and R. E. Allen, in Proceedings of the American Vacuum Society, Topical Conference on Thin Film Processing and Characterization of High $-T_{c}$ Superconductors, edited by J. M. E. Harper, R. J. Colton, and L. C. Feldman, AIP Conference Proceedings No. 165 (Americal Institute of Physics, New York, in press).

${ }^{13}$ B. A. Richert and R. E. Allen, in High Temperature Super- conductors, edited by M. B. Brodsky, R. C. Dynes, K. Kitazawa, and H. L. Tuller [Mater. Res. Soc. Proc. (to be published)]

${ }^{14}$ D. A. Papaconstantopoulos (private communication); O. F. Sankey (private communication).

${ }^{15}$ W. A. Harrison, Electronic Structure and the Properties of Solids (Freeman, San Francisco, 1980).

${ }^{16}$ D. W. Murphy, S. Sunshine, R. B. van Dover, R. J. Cava, B. Batlogg, S. M. Zahurak, and L. F. Schneemeyer, Phys. Rev. Lett. 58, 1888 (1987); R. J. Cava, A. Santoro, D. W. Johnson, and W. W. Rhodes, Phys. Rev. B 35, 6716 (1987); T. Siegrist, S. Sunshine, D. W. Murphy, R. J. Cava, and S. M. Zahurak, Phys. Rev. B 35, 7137 (1987), and references therein.

${ }^{17}$ A. W. Luehrmann, Adv. Phys. 17, 1 (1968).

${ }^{18}$ D. J. Chadi and M. L. Cohen, Phys. Rev. B 8, 5747 (1973).

${ }^{19}$ Y. Chang, M. Onellion, D. W. Niles, R. Joynt, G. Margaritondo, N. G. Stoffel, and J. M. Tarascon, Solid State Commun. 63, 717 (1987).

${ }^{20} \mathrm{G}$. Margaritondo and $\mathrm{M}$. Onellion (private communications).

${ }^{21}$ T. J. Wagener, Y. Gao, J. H. Weaver, A. J. Arko, B. Flandermeyer, and D. W. Capone II, Phys. Rev. B 36, 3899 (1987).

${ }^{22}$ Y. Gao, T. J. Wagener, J. H. Weaver, A. J. Arko, B. Flandermeyer, and D. W. Capone II, Phys. Rev. B. 36, 3971 (1987).

23J. A. Yarmoff, D. R. Clarke, W. Drube, U. O. Karlsson, A. Taleb-Ibrahimi, and F. J. Himpsel, Phys. Rev. B 36, 3967 (1987).

${ }^{24}$ N. G. Stoffel, Y. Chang, M. K. Kelly, L. Dottl, M. Onellion, P. A. Morris, W. A. Bonner, and G. Margaritondo, Phys. Rev. B (to be published). See also M. Onellion, Y. Chang, M. Tang, R. Joynt, E. E. Hellstrom, M. Daeumling, J. Seuntjens, D. Hampshire, D. C. Larbalestier, G. Margaritondo, N. G. Stoffel, and J. M. Tarascon (unpublished). 